\title{
Development and Implantation of Two Teleradiology and Teleconsulting Applications in Catalunya: RAIM and CARE
}

\author{
Octavio Barbero $^{(1)}$, Xavier Catasús ${ }^{(1)}$, Josep Fernàndez ${ }^{(1)}$, Rosa Ruiz $^{(1)}$, Rafael Valls ${ }^{(1)}$ \\ Ricardo Toledo ${ }^{(2)}$, Josep Paradells ${ }^{(3)}$ \\ (1) UDIAT (Unitat de Diagnòstic per la Imatge d'Alta Tecnologia)* \\ (2) UAB (Universitat Autònoma de Barcelona) \\ (3) UPC (Universitat Politècnica de Catalunya)
}

\begin{abstract}
Two teleradiology applications with the added-value of teleconsulting are described: CARE, a high level diagnostic oriented application with videoconference and image and cursor synchronization based on SUN stations, and RAIM, a lower level PC-based application oriented to the transmission of patients' results, reports and associated images. Their technical characteristics and solutions adopted are presented together with the context in which they have been developed and thought to fit in.
\end{abstract}

\section{General context of health care in Catalunya}

Catalunya is placed in the north-east corner of Spain, next to the frontier with France. It has a surface of 32.000 $\mathrm{km}^{2}$ and population of about 6 million inhabitants, 3 million in the metropolitan area of Barcelona (the main city with 1,8 million people).

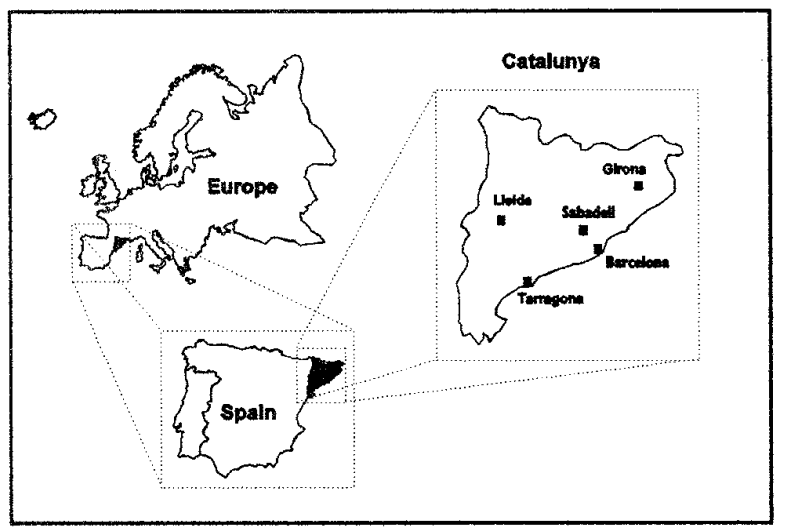

Figure 1. Geographical situation of Catalunya and main cities

" UDIAT, Image Laboratory, Parc Tauli s/n, 08208 Sabadell, Spain
In the last years there is the tendency to medium-sized hospital mergers (hospital consortia) and collaborations for cutting costs down: management, laboratory, radiology departments, etc. So, a more often found situation is having radiological services distributed in several buildings. Also, there is a closer relationship between out-patient and inpatient attendance, and a growing need for reinforcing communication between inter-hospital and intra-hospital departments in all this changing structure. The result is an increasing need for remote access to information (reports and images in our case), and for consultation between distant doctors.

\section{UDIAT: \\ High Technology Imaging Diagnostic Unit}

UDLAT (Unitat de Diagnòstic per la Imatge d'Alta Tecnologia - High Technology Imaging Diagnostic Unit) is an entity of the Department of Health Care and Social Security of Catalunya for the encouragement of basic and applied research on imaging technology and digital communications and for providing access to the use of high technology diagnostic imaging. It is placed in Sabadell, a city of about 200.000 inhabitants and located 30 kilometers far from Barcelona in Spain.

The first aim of UDIAT is the diagnostic support of patients through images, specially MR and CT imaging (it has several MRI and CT systems). UDIAT supports a network of 40 medium-sized hospitals spread all round the Catalan geography with a population of about 3 million inhabitants, except for the metropolitan area of Barcelona.

It is also an aim of UDIAT to develop clinical and technical research programs in order to evaluate new diagnostic imaging techniques, to increase the use of them and to promote processing and transmission of digital biomedical images.

Another objective of UDIAT is to assess the Department 
of Health Care and Social Security of Catalunya, and Catalan hospitals in general on their tasks of planning and politics of investment, use and coordination of high technology imaging.

There are specific needs to cover at UDIAT. After patients have been attended, the information (radiological report and images) has to be returned to their original centers. In a number of cases it has to be returned as soon as possible (emergencies, for instance). Our radiologists consider it essential to send images and radiological information together, using the same physical support. Less important is consulting to our experts from other centers.

\section{RAIM: \\ Medical Imaging Assisted Network}

RAIM (Red Asistida de Imágenes Médicas - Medical Imaging Assisted Network) has been developed by UDIAT together with the Autonomous University of Barcelona (UAB). It runs on PCs or compatible computers and Windows $[1,2,3]$, and so it is a low cost application, easy to use, install and maintain for its graphic interface. RAIM can manage patient information (personal, clinical and radiological) and associated images, display and send all patient data to remote stations together with a written (noninteractive) consultation if necessary. Information is divided into local (input information comes from the local center) and remote patient information (patient data arrived from a distant station). More in detail, this software allows for:

1.- Image acquisition from several image modalities (CT, MR, film digitizers). Images are picked from an image container that receives images generated by MR, CT machines or film digitizers. Image format supported is DICOM [4]: a DICOM compliant application has been developed for film digitizers ( 8 and 12 bits, laser [5] and CCD [6] technologies). No compression is used [7].

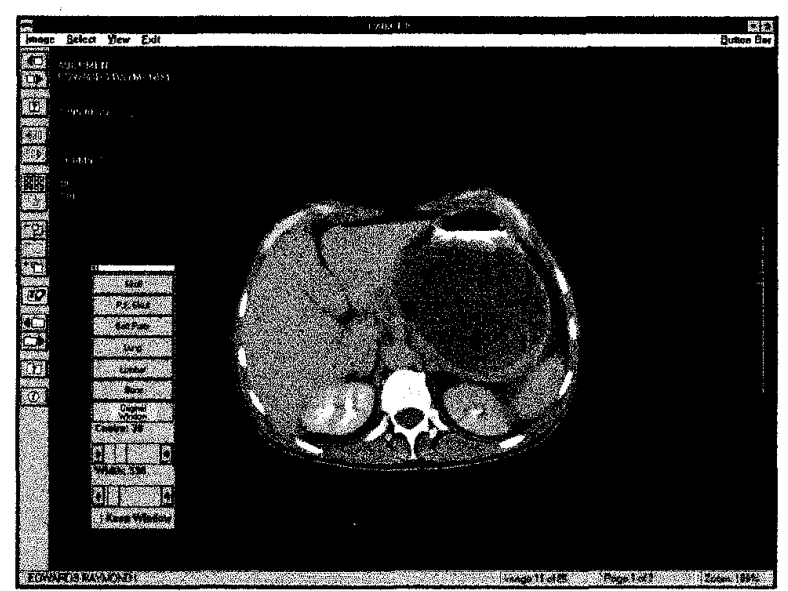

Figure 2. Stack view in RAIM showing the window leveling tool
2.- Database management of patient's information [8] Personal data, clinical and radiological information and images are treated as a whole. Addition, deletion and modification of patients are possible. Tools for the edition of clinical and radiological information are also provided.

3.- RIS integration. Radiological and patient information of the local database is retrieved via FTP standard communications protocol when a RIS is available.

4.- Image display and manipulation[9]. Single or tile image view is available as well as typical handling tools: change from single to tile and vice versa, window leveling (with pre-defined windows for CT images), zooming, display of additional information, change of patient... Easy access to typed patient information (personal, clinical and radiological) and change of patient are also provided.

5.- Transmission of clinical information and radiological reports together with images via ISDN [10] (EuroFile Transfer, Euro ISDN) and LAN-WAN (TCP/IP) to other remote stations. ISDN transmission supports a basic access (one or two B-channels) and telephone in the same board so two doctors can consult a patient while speaking on the phone and sending images at the same time. Local and remote patients are distinguished. Local patients are those whose information has been acquired at the center where the application is being run, and remote patients are those received from other centers. Peer to peer non-interactive consultation is possible for each of the patients.

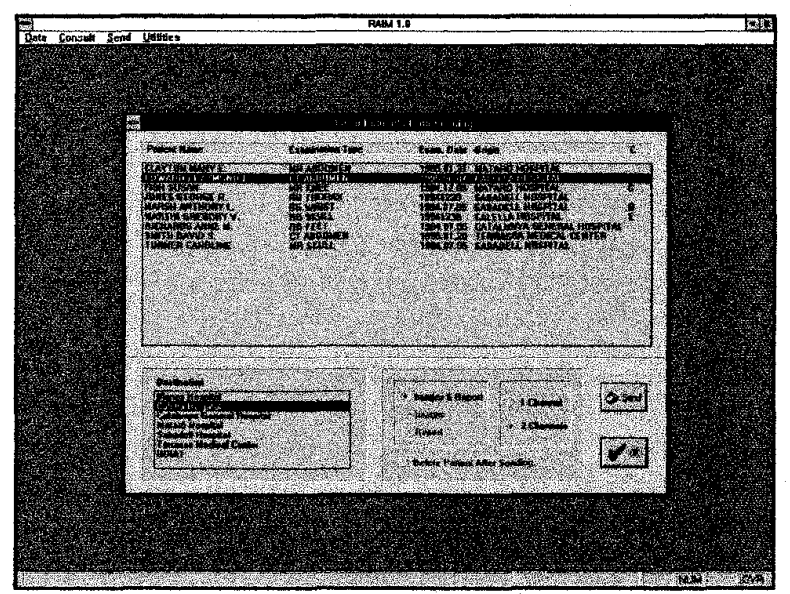

Figure 3. RAIM: Sending of patient information via ISDN

6.- Security access to patient information. An access password for each user is required to execute the application. There are several access levels depending on the type of user: a type of user is able to access personal data and edit them, and another type of user is able to view images and sign radiological reports. The patient's radiological report is encoded so it cannot be edited or viewed by applications other than RAIM. There is the 
possibility of signing radiological reports with a secret sign for each user allowed to. No report can be sent without having been previously signed. Also, after a doctor has signed a report, it cannot be modified. Tools for security maintenance are provided.

At the present time RAIM is in a clinical evaluation phase at UDIAT in collaboration with other Catalan hospitals. It starts to be used at hospitals with their radiological service in more than one building, a couple of hospitals with collaboration agreements on service sharing, and at UDIAT for distributing images to some of the 40 health care services attended.

\section{CARE:}

\section{Computer Assisted Radiology Environment}

CARE project has been developed by UDIAT's Image Laboratory, together with two universities (Universitat Autònoma de Barcelona and Universitat Politècnica de Catalunya). It is a high level teleradiology [11] and telepresence system based on SUN workstations and XWindows/Solaris 2.x [12, 13], so it is easy to use and maintain due to its graphic interface. This software allows for image acquisition from several image modalities, database management of patient information, multimedia instruments (voice, text, etc.) and communication; associated display tools have been developed in order to help diagnostic. Communication can be established in two different ways: non interactive consultation (transmission of non-real time information) like electronic mail, and interactive consultation (transmission of real time information) with videophony and image and cursor synchronization. The main features of CARE are better explained in the following points:

1.- Image acquisition. $\mathrm{CT}, \mathrm{MR}$, film digitizers are acquired from a DICOM image container where images have

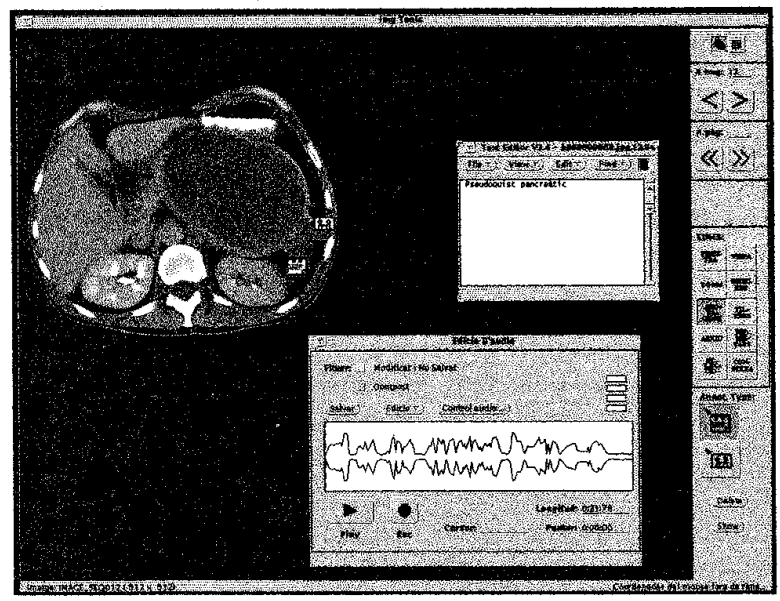

Figure 4. Stack view in CARE showing text and audio annotations to be previously placed. Images are transferred from the image container and incorporated in the information structure of the program. A DICOM compliant application has been developed for film digitizers ( 8 and 12 bits, laser and CCD). FTP is used to transfer images from the container to the system by means of an acquisition server which receives messages indicating which patient has to be acquired.

2.- Patient information manipulation. Two types of patients are distinguished: local patients, those who have been acquired at the local system, and remote patients, those received from remote stations. Information is structured in three levels: patients (local and remote), studies, each patient having several studies, and examinations (several for each study)

3.- Image display and handling [14]. Images can be viewed individually or in a tile of images. There are diagnostic oriented tools like measures (distances, angles), zoom, window leveling (12 bit images), bright and contrast adjustment, marking of zones of interest, attachment of voice and text annotations on specific points of images, flipping and rotation, filters, etc. Voice and text noninteractive consultation of selected images is provided.

4.- Non-interactive consultation. This means that voice and text consultations can be sent to remote stations in a similar way to electronic mail (non-real time consultation). This can be done at the following levels of information: study (all examinations are sent together with text or voice), examination (all images of the selected examination are sent), and selected images (only selected images on the screen are sent). TCP/IP (TCP/IP on ISDN also provided) is the communications protocol used.

5.- Interactive consultation. It is possible to establish a connection using videoconference and image and cursor synchronization. A previous non-interactive consultation must have been carried out before an interactive

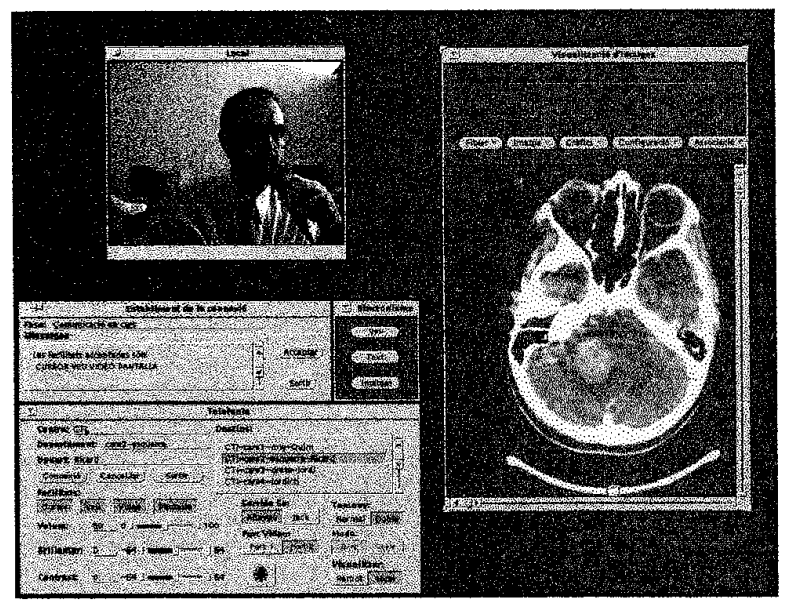

Figure 5. CARE: A view of an interactive consultation 
consultation. When connecting with a remote station, all common consultation are checked and presented to the originator partner. Selecting a patient starts the consultation. Videoconference allows to see and talk to the remote partner through a window displayed on the screen. This window can also be switched to local and receive video coming from either a first video port (usually connected to the local camera) or a second port (echograph, video cassette recorder, etc). There is a control of master to allow taking control of the images being displayed. Image synchronization means both partners see exactly the same images on the screen and the same operations (zoom, bright and contrast, filters and the like) are performed at both sites. This operations are performed by the master cursor (a tool for getting control of the connection is provided). Cursor synchronization means that both sites can see the other partner's cursor movements. This is useful, for instance, for marking zones of interest while discussing about them.

CARE has been evaluated within the European project TRILOGY. A general good impression was obtained from radiologists who participated in terms of quality (sound and video, image display and tools), but several changes in the user interface have been made, resulting in a very easy-touse interface. At the present time CARE is in a precommercial phase. IBERMATICA, one of the main Spanish computer enterprises (IBM is the majority shareholder), is in charge of its commercialization. The main difficulty to introduce CARE is that, in general, communication priorities point at other directions rather than videoconference: connection with HIS (EDIFACT standards are being considered).

\section{Conclusions}

Having a look at the general context of health care in Catalunya and the needs to cover, particularly at UDIAT, one can see the features that identify teleradiology and teleconsulting as the most appropriate solutions for those necessities: transmission of radiological reports and associated images, remote display of this images and access to reports, consultation between doctors (experts) between distant centers or within a distributed radiological department. RAIM and CARE, as teleradiology and teleconsulting applications, were designed to cover these needs. RAIM, as running on PCs, is more affordable than CARE, and has been thought as a tool for sending images to remote stations, image viewing and access to reports (editing, signing, etc). CARE is a diagnostic oriented application running on SUN stations for teleconsultation with some very helpful features: videoconference, image and cursor synchronization, etc.

Future evolution will be to scale RAIM up to CARE (diagnostic-oriented tools, text and voice annotations, teleconsultation with videoconference), HIS integration with regard to EDIFACT standards, and other medical utilities coming from daily use and evaluation processes, some of which will be realized within projects of the Telematics Applications Programme of the IV Framework Progranme of the European Union.

\section{References}

[1] Petzold, Charles. Programming Windows 3.1. Microsoft Press. 1992

[2] Borland C++4.5 Manuals, Borland International, Inc., 1994

[3] Jackson M. System Development. Englewood Cliffs, NJ: Prentice Hall. 1983.

[4] Digital Imaging and Communications in Medicine (DICOM), Final draft, ACR-NEMA Standards Publication PS3.x, 1993.

[5] Lumiscan 50 Software Library Reference Guide, Lumisys, Inc, August 1993.

[6] VIDAR SCSI Toolkit Manual, Vidar Systems Corporation, January 1995.

[7] Chen J, Flynn MJ, Gross B, Spizarny D. Observer detection of image degradation caused by irreversible data compression processes. Proc SPIE 1991; 1444: 256-264.

[8] Stein J. Object-Oriented Programming and Database Design Dr. Dobb's Journal of Software Tools for the Professional Programmer, $\mathrm{N}^{\circ} 137$, p. 18.1988.

[9] Gur D, Experience with CRT displays in the radiology department. Presented at the Society for Information Display 1991 International Symposium, Seminar and Exhibition, Anaheim, Calif. May 6-10 1991. Proc SID 1991; 353-354.

[10] EUROFILE-DDE-SERVER, Teles GmbH.

[11] Elliot, Larry P., Mun, Seong K., Horii, Steven C., Benson, Harold. Digital Imaging Network System. Evaluation Report. Department of Radiology, Georgetown University Medical Center.

[12] Solaris 5.x reference manuals. Sun Microsystems Inc.

[13] X-Windows Reference Manuals: O'Reilly series, Vol 0 to 7.

[14] Good WF, Burzik CM, Scanlon PJ, Maitz GS, Herron JM, Gur D. User Friendly electronic film library for digital imaging modalities. European Radiol 1992. 